\title{
MedienPädagogik
}

Zeitschrift für Theorie und Praxis der Medienbildung

www.medienpaed.com

ISSN 1424-3636

Jahrbuch Medienpädagogik 15:

Erziehungswissenschaftliche und medienpädagogische Online-Forschung:

Herausforderungen und Perspektiven

Herausgegeben von Johannes Fromme, Stefan Iske, Therese Leik, Steffi

Rehfeld, Jasmin Bastian, Manuela Pietraß und Klaus Rummler

\section{Situationsanalyse in der medienpädagogischen Mediatisierungsforschung}

Kommunikative Figurationen des informellen Lernens

\author{
Karsten D. Wolf und Konstanze Wegmann
}

\section{Zusammenfassung}

Der Beitrag fragt nach den Konsequenzen der kommunikationswissenschaftlichen Mediatisierungstheorie für die medienpädagogische Theorieentwicklung und Forschung. Zentral dabei ist die Forderung, nicht mehr den Einfluss von Einzelmedien zu untersuchen, sondern vielmehr auf einer Meta-Ebene zu analysieren, wie sich unser kommunikatives Handeln und die sich daraus konstruierenden Handlungssituationen innerhalb einer zunehmend komplexeren Medienumgebung verändern. Als theoretische Basis und empirischer Analyseansatz wird dazu zunächst das Konzept der Kommunikativen Figurationen vorgestellt; im Anschluss wird die Situationsanalyse als geeignete Methode zur Rekonstruktion kommunikativer Figurationen vorgeschlagen und ihre Potenziale für die medienpädagogische Forschung anhand einer Beispielstudie zu den kommunikativen Figurationen des informellen Lernens verdeutlicht. Der Artikel soll so einen Beitrag zur medienpädagogischen Theorieentwicklung sowie zum medienpädagogischen Methoden-Diskurs leisten. 


\title{
Situational Analysis in Media Educational Mediatization Research: Communicative Figurations of Informal Learning
}

\begin{abstract}
This article discusses consequences of mediatization theory for theory development and research in the field of media education. The central approach of mediatization is to analyse on a meta level, how our communicative action and the thereby constructed situations of action transform within an increasingly complex media environment, rather than to focus on the research on one single medium. First, communicative figurations are introduced as an analytical concept for opening these media-related changes for analysis. Second, Situational Analysis is proposed as a method for the reconstruction of communicative figurations. Finally, the method's potentials for research in media education are illustrated using an exemplary study of the communicative figurations of informal learning. This article aims to contribute to theory development in the field of media education as well as to the methodological discourse of media education research.
\end{abstract}

\section{Einleitung - Mediatisierung und ihr Bezug zur medienpädagogischen Theorieentwicklung}

Wir leben in einer Zeit, in welcher der technische Fortschritt und dessen Veränderungsgeschwindigkeit im Alltag besonders offensichtlich bei den (digitalen) Informations- und Kommunikationstechnologien hervortreten. So konstatiert Benjamin Jörissen, dass «Digitalisierung und mobile Vernetzung (...) unsere Lebenswelten und ebenso die Lebenswelten von Kindern und Jugendlichen enorm verändert» (Jörissen 2015, 101) haben. Dieser laufende Veränderungsprozess wird in Teilen der Kommunikations- und Medienwissenschaft (KMW) als Mediatisierung erforscht. Mediatisierung ist allerdings nicht als Beschreibung einer zunehmenden Verbreitung von technischen Medien in der Gesellschaft und deren Beeinflussung 
durch diese zu verstehen, sondern stellt eine Meta-Theorie dar, mit Hilfe derer wir erforschen können, wie sich unser kommunikatives Handeln und die sich daraus konstruierenden sozialen Handlungssituationen durch Medien verändern; gleichzeitig aber auch, wie diese Medien im Wechselbezug durch Aneignung ${ }^{1}$ umgeformt werden (Krotz 2007; Couldry und Hepp 2016). Mediatisierung ist ein Prozess, der weit in die Anfänge der Moderne zurückverfolgt werden kann und nicht linear, sondern widersprüchlich und lokal verschieden verlaufen kann (Hjarvard 2013; Lundby 2014; Meyen 2009; Thompson 1995).

Die Mediatisierungstheorie vermittelt zwischen den bisherigen Extrempositionen der KMW «Wie wirken Medien auf Menschen» vs. «Was machen Menschen mit Medien» (Hjarvard 2013, 2) auf unterschiedlichen Ebenen:

- Makroebene: Medien beeinflussen unsere kommunikativen Praktiken, Haltungen und Einstellungen in zunehmendem Masse. So hat z.B. das Smartphone als kombiniertes Fotografie- und Vernetzungstool in Kombination mit Diensten wie Instagram zu einer neuen Form und Intensität der Selbstdarstellung (nicht nur) Jugendlicher geführt;

- Mikroebene: Menschen beeinflussen die Gestaltung und Weiterentwicklung von Medien durch ihre individuelle Aneignung. Sie sind den Medien nicht ausgeliefert, sondern verfolgen ihre eigene Agenda, z.B. durch einen kreativen Misuse der Medien, welcher dann von den Medienproduzierenden aufgenommen wird. So hatten Mobiltelefone zunächst nur eine Kamera eingebaut. Durch das Aufkommen von Selfies wurde aber die Frontkamera

1 Der Begriff der «(Kommunikativen) Aneignung» bezeichnet den Vorgang des «Sich-zu-Eigen-Machens» von Medieninhalten/-produkten (spezifischer «Medienaneignung») oder anderen Produkten, Alltagserfahrungen etc. in Abgrenzung zu Begriffen wie "Konsumption», die eher von eindimensionalen Wirkungen von (Medien-)inhalten/-produkten auf Menschen ausgehen (vgl. zusammenfassend Hepp 2005). 
zu einem zunehmend unverzichtbaren Element moderner Smartphones. Aber auch durch das direkte Feedback ihrer Nutzung z.B. durch Klick-Zahlen oder Uploads bestimmen sie Inhalte, Funktionen und «Logiken» mit. Dies betrifft weniger die klassischen Massenmedien, sondern vielmehr die partizipativen Medien des Internets bzw. der Mobilkommunikation;

- Mesoebene: Der Begriff der Mediatisierung zielt im Kern auf die wechselseitige Beeinflussung von Medien, Kultur und Gesellschaft: Medien sind nahezu überall und durchdringen alle soziale Sphären wie z.B. die Politik, die Religion, aber auch die Bildung. Ebenso wie auf der Mikroebene sind Medien dabei nicht als zentrale Agenten des Wandels zu verstehen, sondern als ein Systemelement, welches beachtet und in Theorien integriert werden muss, um kommunikative - und damit alle sozialen - Prozesse zu analysieren, zu verstehen und zu modellieren. Gleichzeitig sind Medien aber so tief in unsere Gesellschaft und Kultur integriert, dass ein analytisches Herauslösen nicht mehr möglich erscheint.

Diese miteinander verschränkte Perspektive erzeugt eine hohe Passung zu den Erkenntnisinteressen einer handlungsorientierten Medienpädagogik (sensu Baacke 1973, 1999; Tulodziecki 2007; Hugger 2012; vgl. auch den Entwurf einer integralen Medienpädagogik von Schorb 2011, 92ff.; zur Dialektik zwischen Medienerziehung und Medienaneignung in der Medienbildungsdebatte vgl. Wolf et al. 2011): Aus der Perspektive der Medienpädagogik gilt es, sowohl dysfunktionale Einflüsse als auch ermächtigende Potentiale von Medien auf der Makroebene zu identifizieren, um auf der Mikroebene die Individuen im Sinne einer Subjektivation (Butler 2001; Jörissen 2017) dabei zu unterstützen, die notwendigen (Medien-)Kompetenzen aufzubauen, um Medien für die eigenen Ziele erfolgreich einzusetzen bzw. zu verändern. Die Mediatisierungstheorie ist für die Medienpädagogik deshalb so wichtig, weil sie ihr einen Theorierahmen zur Verfügung stellt, mit dem erziehungs- und bildungswissenschaftliche Fragen zu den Aushandlungsprozessen auf der oben beschriebenen Mesoebene erforscht und analysiert werden können. 
Ziel des Artikels ist es, vor dem Hintergrund der kommunikationswissenschaftlichen Mediatisierungstheorie, die Potenziale der Situationsanalyse für den medienpädagogischen Methoden-Diskurs herauszustellen. Zur Verdeutlichung der Potenziale der Situationsanalyse, sowie des Ansatzes der kommunikativen Figurationen, für die medienpädagogische Forschung wird eine eigene Beispielstudie zu den kommunikativen Figurationen des informellen Lernens herangezogen. Im Folgenden wird zunächst auf die Grundelemente der Mediatisierungsforschung eingegangen.

\section{Grundelemente einer Mediatisierungsforschung}

In der KMW werden - wie auch in der Mediensoziologie - zunehmend Ansätze verfolgt, welche die sozialen Praktiken sowie die Handlungsmöglichkeiten der Individuen im Sinne des Agency-Paradigmas untersuchen (Sewell 1992). Sowohl in der Publikumsforschung (Blumler und Katz 1974; Renckstorf und Wester 2001; Teichert 1972) als auch in den Cultural Studies (Certeau 2002; Hall 1973) wurden seit Anfang der 1970er Jahre Mediennutzende nicht mehr als willfährige und von den Medien gesteuerte Objekte betrachtet, sondern als handelnde und reflexionsfähige Personen. Dies geht einher mit dem ersten Medienkompetenzmodell, welches von Dieter Baacke als Gegenentwurf sowohl zur Bewahrpädagogik als auch zur kritisch-materialistischen Medienpädagogik entworfen wurde (Baacke 1973). Heute werden sowohl die Medienproduzierenden als auch die Mediennutzende als Akteurinnen und Akteure verstanden, welche mit Medien aktiv umgehen (Bonfadelli und Friemel 2014; Hasebrink 2003; Napoli 2010; Neumann-Braun 2005). Spätestens mit dem Aufkommen des Social Webs, aber eigentlich bereits mit der Demokratisierung von Produktionsmitteln wie dem Kassettenrekorder, dem Super-8-Film oder Videokameras, hat sich die klare Trennung zwischen Produzierenden und Konsumierenden aufgelöst, wie es Bruns mit dem Konzept des Produtzers (Produzent und Nutzer) beschreibt (Bruns 2008; Bruns und Schmidt 2011). 
Solch eine auf die Medienpraktiken fokussierte Forschung folgt überwiegend einem praxeologischen Ansatz (Schatzki et al. 2001), in dem die körperlich ausgeführten Praktiken bestimmend für die Konstitution sozialer Welten sind. Das Interesse liegt dabei auf dem «embodied doing», also dem in der physikalischen Gesamtheit des Körpers erlebten Tun. Dieses Tun wird nach Giddens (1984) hochkontextualisiert und häufig unbewusst in komplexen Handlungssituationen erlernt. Dieses praktische und überwiegend implizite Handlungswissen ist den Handelnden gerade nicht diskursiv erschliessbar, formt aber einen elementaren Teil des Habitus einer Person (Bourdieu 1992). Kommunikationspraxen sind somit verleiblichte («embodied») Praktiken der Mediennutzung (Couldry 2004, 125). Aus einer sozialkonstruktivistischen Sicht heraus (Bongaerts 2007) schliesslich können wir diese als komplexe und hochkontextualisierte Handlungsmuster verstehen. Die Bedeutung von Kommunikationspraktiken hängt von sozialen Vereinbarungen ab und konstruiert die menschliche Realität bzw. ihre soziale Welt (Keppler 2005; Knoblauch 2013). Dieser praxeologische Turn der Sozial- und Kulturwissenschaften ermöglicht es, den wechselseitigen Prozess der Durchsetzung von Medienlogiken im Sinne der direkten und indirekten Mediatisierung nach Hjarvard (2013) (Metaebene) mit der Aneignung bzw. «Domestizierung» (Silverstone 2006) durch die Individuen (Mikroebene) in Verbindung zu setzen.

Kommunikationsmedien institutionalisieren, objektivieren und materialisieren Symbolsysteme und (Kommunikations-)Praktiken (Berger und Luckmann 1967; Couldry und Hepp 2016; Fornäs 2000; Knoblauch 2013). In diesem Sinne formen Medien durch ihre spezifischen Handlungsmuster Kommunikation. Vor dem Hintergrund einer tiefgreifenden Mediatisierung ${ }^{2}$ stellt sich deshalb die Frage, welchen Einfluss diese Medien im Kontext komplexer Medienkonstellationen auf unsere Kommunikationsprozesse und damit auf die

2 Unter dem Begriff «tiefgreifende Mediatisierung» («deep mediatization») verstehen Hepp und das CoFi Research Network (2017) eine insbesondere durch Digitalisierung vorangetriebene - Intensivierung des Prozesses der Mediatisierung. 
soziale Konstruktion der Wirklichkeit haben und welche Bedeutung dies für Bildungsprozesse hat.

Dies kann auf drei Ebenen betrachtet werden: auf der Ebene der Medienumgebung, welche die aktuelle Gesamtheit aller zur Verfügung stehenden Medien darstellt; der Ebene der Medienensembles, welche die in einer «sozialen Domäne» ("social domains», Hjarvard 2013, 17; siehe auch Hepp und Hasebrink 2017, 332f.) wie z.B. der Familie, der Schule oder der Arbeit genutzte Teilmenge der Medienumgebung beschreibt; schliesslich auf der Ebene des Medienrepertoires, welche die Auswahl der Medien meint, die ein Individuum in seinen alltäglichen Praxen einsetzt und sich aneignet (Hasebrink und Popp 2006; Hasebrink und Domeyer 2012). Dabei reichen diese Kommunikationspraktiken auf allen Ebenen über eine Vielfalt von Medien hinweg. Wenn wir z.B. für eine Klausur lernen, nutzen wir eigene schriftliche Aufzeichnungen und mit dem Smartphone gemachte Fotos von Folien aus Vorlesungen, Bücher, eBooks, PDFs, Videoaufzeichnungen von Vorlesungen, Online-Skripte, YouTube-Tutorials, Lernsoftware und viele andere Medienformen. Diese Vielfalt und ihre gegenseitigen Einflüsse sind in einer Analyse unserer Kommunikationsprozesse zu berücksichtigen.

Typisch für Forschungsprojekte im Mediatisierungsparadigma sind qualitative Methodenansätze, welche die u.a. in Einzel- und Gruppeninterviews, Medientagebüchern, visuellen Artefakten, Online-Texten, wie z.B. Kommentaren oder Blogbeiträgen, sowie ethnografischen Studien gewonnenen Daten im Sinne der Grounded Theory oder mittels der qualitativen Inhaltsanalyse sowie der kritischen Diskursanalyse auswerten. Häufig werden Datenquellen im Sinne von Multi-Site-Studien kombiniert, ebenso Forschungsmethoden im Multi-Method-Design kombiniert und deren Daten trianguliert. Schliesslich ist auch die Integration quantitativer Forschungsmethoden denkbar, z.B. um die Durchdringung von Medien in den Medienrepertoires einer grösseren Benutzendengruppe mit standardisierten Befragungen zu erheben. 
Vergleichbar zu subjektorientierten medienpädagogischen Forschungsansätzen (Schlör 2017) übernehmen Interviewpartnerinnen und -partner dabei eine durchaus gestaltende Rolle. So werden zur Bestimmung der Kommunikationsrepertoires in themenzentrierten Interviews 《qualitative Netzwerkkarten» von den Interviewten zunächst selbst angefertigt, auf denen die Medien, Kommunikationspartnerinnen und -partner und Kommunikationsfunktionen ähnlich einer Concept Map visualisiert werden, um dann im Interview zur weiteren Erörterung genutzt zu werden (Hepp et al. 2012, 89ff.). Diese zunächst eigenerstellten Visualisierungen, welche wir hier in Anlehnung an die weiter unten vorgestellte Situationsanalyse als «messy communicative repertoire maps» bezeichnen, können für die weitere Auswertung auf Basis der qualitativen Auswertung strukturiert und geordnet werden («ordered communicative repertoire maps»).

Um für das umfassende Konzept der Mediatisierungstheorie und -forschung nun einen konkreten Analyseansatz zu bieten, schlagen wir im Folgenden das Konzept der Kommunikativen Figurationen vor und zeigen Möglichkeiten ihrer Erforschung auf.

\section{Kommunikative Figurationen und ihre Erfor- schung}

Abweichend von bisherigen Ansätzen in der KMW zielt das vom Communicative-Figurations-Research-Network entwickelte Forschungsparadigma auf eine nicht-medienzentrierte Analyse kommunikativer Prozesse unter besonderer Berücksichtigung der Medien (Hepp und CoFi Research Network 2017; Moores 2012; Morley 2009; Krajina et al. 2014). Analyseeinheiten sind soziale Domänen. Diese dienen nach Hepp und Hasebrink als «weiterer Oberbegriff für verschiedenste soziale Zusammenhänge, für 〈sinnhafte Bereiche〉 der Gesellschaft» (Hepp und Hasebrink 2017, 333). Diese Bereiche skalieren «von Paaren, Gruppen, Gemeinschaften und Organisationen 
bis hin zu ganzen sozialen Feldern» (ebd., 333). Soziale Domänen umfassen u.a. Konzepte wie soziale Felder (Bourdieu 1993), (Sub)Systeme (Systemtheorie; Luhmann 2012, 4ff.), (kleine) Lebenswelten (Sozialphänomenologie; Luckmann 1970, 587; Schütz 1967, 139ff.) oder soziale Welten (Symbolischer Interaktionismus; Clarke 2011a, 384f.; Shibutani 1955, 566).

Bezogen auf Bildung können soziale Domänen also ganze Schulsysteme oder das System der beruflichen Ausbildung sein, aber auch Schulen, Nachhilfeanbietende, Klassen und deren Peer-Vergemeinschaftungen sowie die am Ende des Beitrages beschriebenen Mediatisierungsvergemeinschaftungen des informellen Lernens. Wichtig für die Analyse der Kommunikationsprozesse ist, dass die Akteurinnen und Akteure in den jeweiligen sozialen Domänen aufeinander bezogene Praktiken teilen. Jede soziale Domäne besitzt deshalb eine typische «Akteurskonstellation» (Schimank 2010, 202ff.), in der alle relevanten Akteurinnen und Akteure sowie ihre Beziehungen zueinander beschrieben werden.

Was heisst das für medienpädagogische Forschungsprojekte? Ziel unserer theoretischen und empirischen Auseinandersetzung mit dem Konzept der Kommunikativen Figurationen soll es sein, die Veränderung bildungsbezogener sozialer Domänen nachvollziehbar zu machen. Es geht also nicht darum, einseitig die Wirkung von z.B. YouTube-Tutorials auf die Lernpraxis von Schülerinnen und Schülern zu untersuchen, sondern vielmehr darum, 1) wie sich die Kommunikationspraxen des schulbezogenen Lernens von Schülerinnen und Schülern im Kontext einer tiefgreifenden Mediatisierung verändern, indem sie z.B. in WhatsApp-Gruppen Lösungen teilen, im Internet recherchieren, YouTube-Tutorials schauen und auf Lernplattformen Übungsaufgaben lösen; 2) welche Konsequenzen dies wiederum für das soziale Subsystem Schule und die Gestaltung von Klassenarbeiten, Unterricht und Leistungsüberprüfungen hat; 3) welche Veränderungen die Medien dabei erfahren, um sich z.B. den ausformenden Kommunikationspraxen anzupassen und neue Funktionalitäten und Geschäftsmodelle zu entwickeln. 
Diese Netzwerke kommunikativer Praktiken werden von der Bremer und Hamburger Forschungsgruppe in Rückgriff auf den prozess-soziologischen Ansatz von Norbert Elias (1978) als kommunikative Figurationen bezeichnet (Hepp und CoFi Research Network 2017). Das Elias'sche Konzept der Figuration entstand aus seiner Auseinandersetzung mit zwei grundlegenden Problemen soziologischer Analyse: zum einen der Autonomie des Einzelnen bei gleichzeitiger Kodependenz (Gegenabhängigkeit) von Individuum und Gesellschaft, sowie der Unterscheidung zwischen sozialem Wandel und strukturellem Wandel. Nach Elias kann jede strukturelle Veränderung als ein sich transformierender Zusammenhang zwischen Individuen und Gesellschaft verstanden werden. Diese dynamischen Relationen bezeichnet er als Figurationen, die für ihn «Netzwerke von Individuen» sind (Elias 1978, 15). Aus einer kommunikations- und medienwissenschaftlichen Perspektive beschreiben wir mit kommunikativen Figurationen die kommunikativen Konstruktionsprozesse sozialer Wirklichkeit innerhalb beliebiger sinnhafter Bereiche der Gesellschaft durch spezifische Akteurskonstellationen und Medienensembles. Kommunikative Figurationen können dabei sowohl die Perspektive von Individuen, Vergemeinschaftungen als auch Organisationen umfassen. Wenn wir als Beispiel die Vergemeinschaftung der Familie betrachten und deren crossmediale Handlungspraxen beschreiben, wie z.B. die Nutzung des Smartphones und verschiedener Apps, das Festnetztelefonat mit den Grosseltern oder handschriftliche Nachrichten am Kühlschrank, sowie deren Akteurskonstellation, wie z.B. die Mitglieder von Patchworkfamilien und ihre wechselseitigen Kommunikationsbezüge, so ist zu erkennen, dass diese kommunikativen Praxen zentral für den Zusammenhalt der familiären Strukturen sind (Hasebrink 2014).

Zusammenfassend lässt sich jede kommunikative Figuration wie folgt beschreiben (Hepp und Hasebrink 2017):

1. Strukturelle Basis jeder kommunikativen Figuration ist eine Akteurskonstellation, ein Netzwerk von Akteurinnen und Akteure, die in einer bestimmten Machtbalance und durch aufeinander 
bezogene kommunikative Praktiken wechselseitig miteinander verbunden sind.

2. Jede kommunikative Figuration ist gekennzeichnet durch einen Relevanzrahmen, der handlungsleitend für die Praktiken seiner Akteurinnen und Akteure sowie deren wechselseitige Ausrichtung aufeinander ist. Dieser Relevanzrahmen definiert das «Thema» und entsprechend die Sinnorientierung der kommunikativen Figuration.

3. Kommunikative Figurationen werden konstituiert durch kommunikative Praktiken, die verwoben sind mit weiteren sozialen Praktiken und sich auf ein Ensemble verschiedener Medien stützen.

Aus einer medienpädagogischen Forschungsperspektive können wir festhalten: die Mediatisierungstheorie fügt ein Bindeglied zwischen der Makroebene (Medien, Vergemeinschaftungen, Organisationen) und der Mikroebene (Individuum, Subjekt) ein, welches die Analyse der wechselseitigen kommunikativen Konstruktion lernund bildungsbezogener Prozesse ermöglicht. Für aktuelle medienpädagogische Fragen nach einer diskursiven Vermittlung zwischen Gesellschaft, Medien und Individuum (Subjektivation) ist dies ein besonders vielversprechender Ansatz. Die Situationsanalyse nach Adele Clarke scheint hier eine geeignete Methode zur Erfassung, Analyse und visuellen Aufbereitung von kommunikativen Figurationen.

\section{Situationsanalyse nach Adele Clarke}

Aus einer forschungsmethodologischen Perspektive kann man in medienpädagogischen Forschungsprojekten zu kommunikativen Figurationen des Lernens und der Bildung an der in Mediatisierungsstudien häufig verwendeten Grounded Theory Methodologie (GTM) anschliessen. Wie wir hier im Folgenden herausstellen möchten, eignet sich aus unserer Sicht insbesondere eine Weiterentwicklung der Grounded Theory (GT), nämlich die Situationsanalyse nach Adele 
E. Clarke (Clarke 2005), welche die GT um zentrale Aspekte erweitert. ${ }^{3}$ Historisch betrachtet war die GT der erfolgreiche Versuch, im Kontext eines quantitativen Forschungsparadigmas der Soziologie der 1960er Jahre eine empirisch-qualitative Forschungsmethode zu etablieren. Ein besonderes Anliegen war es, die damals vom quantitativen Forschungsestablishment den qualitativen Methoden vorgeworfene Subjektivität hermeneutischer Deutungen durch systematische Datengewinnung und Analysemethoden zu entkräften sowie die induktiven/abduktiven Prozesse der Theoriebildung zu dokumentieren und nachvollziehbar zu machen. Forschung im Stil der GTM fokussiert insbesondere auf soziale Prozesse, nutzt als Daten überwiegend Interviews, Beobachtungen und Feldnotizen, welche über zu entwickelnde Kodiersysteme abstrahiert und systematisiert werden, um schliesslich kleinteilig geschnittene Theorien (bezogen auf die untersuchten Daten) zu entwickeln (Corbin und Strauss 2015). Durch die Zusammenführung einer grösseren Anzahl von GT-Studien mit ähnlichen Erkenntnisinteressen können formalere Theorien mit einer grösseren sozialen Reichweite entwickelt werden. Besondere Merkmale der GT sind - ähnlich wie beim Design Based Research (Anderson und Shattuck 2012; Koppel 2017; Reinmann 2017) - ein iteratives und überwiegend exploratives Vorgehen, in dessen Verlauf die jeweilige Theorie entwickelt wird und gleichzeitig weitere bisher fehlende Analyseeinheiten identifiziert werden. GT ist also weniger als ein eng definiertes methodisches Inventar zu verstehen, sondern vielmehr als ein Forschungsstil.

Wieso nun eine neue Methode? Adele Clarke studierte und arbeitete seit den 1980er Jahren bei Anselm Strauss in den Pflegewissenschaften an der UCSF und übernahm später seinen Lehrstuhl. Dort integrierte sie neben konstruktivistischen und interaktionistischen Perspektiven zunächst feministische und später auch postmoderne,

3 Adele Clarke war Schülerin von Anselm Strauss, der einer der Begründer der GT war (Glaser und Strauss 1967). Strauss war Schüler von Herbert Blumer, der den symbolischen Interaktionismus begründete (Blumer 1969). Blumer bezog sich dabei auf seinen akademischen Lehrer George Herbert Mead. 
poststrukturalistische und interpretative Perspektiven in ihre Forschungsarbeit und entwickelte aus der folgenden Auseinandersetzung mit der GTM die Situationsanalyse (Clarke 2005; Clarke et al. 2018). Knapp zusammengefasst verfolgt sie damit die folgenden fünf Hauptziele:

\subsection{Rekonstruktion von Situationen}

Das zentrale Untersuchungsziel der Situationsanalyse ist die Rekonstruktion der Komplexität von Situationen. ${ }^{4}$ Dabei löst Clarke die Dichotomie von Situation und Kontext auf, welche sich in der GT noch in der «Bedingungsmatrix» finden lässt. Sie argumentiert, dass die Bedingungen der Situation in der Situation seien und diese so von innen heraus konstituieren würden, statt sie von aussen zu beeinflussen (Clarke et al. 2018). Im postmodernen Theoriebezug stellt sie Komplexität, also z.B. Widersprüche, Heterogenitäten und Situiertheit, im Gegensatz zu klaren Kausalitätsbezügen heraus. Somit löst sie sich vom Kodierparadigma des axialen Kodierens der GTM und integriert in ihrer eigenen Version der situativen Bedingungsmatrix - der Situationsmatrix - zwei zentrale neue Aspekte der Situationsanalyse, nämlich Diskurse und nicht-menschliche Aktanten.

\subsection{Einbezug von Diskursen}

Als weiteren Weg der Analyse struktureller Prozesse neben der genannten «Bedingungsmatrix» entwickelten Strauss (z.B. 1978) und weitere die Sozialen Welten/Arenen Theorie, die Strauss selbst jedoch nicht mit der GT verknüpfte. Clarke versteht die Situationsanalyse daher als eine methodologische Weiterentwicklung der GT und Sozialen Welten/Arenen Theorie als solche sowie der Integration

4 Clarke bezieht sich auf das Situationskonzept des symbolischen Interaktionismus nach Herbert Blumer, der eine Situation als den Moment definiert, in dem die teilnehmenden Personen die jeweiligen Bedeutungen gemeinsam aushandeln (Blumer 1973, 84). Die Situation wird also erst durch Interaktionen der Teilnehmenden konstituiert, sie existiert nicht unabhängig von den Akteurinnen und Akteuren. 
beider (Clarke et al. 2018). Sie arbeitet zudem einige Parallelen zwischen Strauss' Soziale Welten/Arenen Theorie und der Diskurstheorie Foucaults heraus. Foucault geht davon aus, dass Macht in Form von Diskursen zirkuliere und durch die alltäglichen Praktiken der Menschen - z.B. durch ihr Handeln gemäss den Vorstellungen einer gesellschaftlichen Norm («technology of the self», Foucault 1988) reproduziert und so in seiner Wahrheit und scheinbaren Natürlichkeit immer wieder bestätigt und verfestigt werde. Individuen sowie Kollektive würden durch Diskurse konstituiert werden, indem sie selbst aktiv gemäss diskursiv zirkulierenden Vorstellungen handeln würden (ebd.). Clarke et al. (2018) argumentieren, dass Diskursformationen wie auch soziale Welten aus widersprüchlichen Diskursen bestehen würden, die dort permanent ausgehandelt, gewertet und positioniert werden. Sowohl Diskursformationen als auch soziale Welten können demnach als Zusammenschlüsse von machtvolleren bzw. einflussreicheren und weniger machtvollen Personen, die gewisse Interpretationen, Werte und Normen teilen, verstanden werden. Als Konsequenz dieser Überlegungen erweitert Clarke die GTM im Rahmen der Situationsanalyse explizit um den analytischen Einbezug von Diskursen und die Analyse von Diskurspositionen. Wie auch Foucault - verdeutlicht durch seinen Begriff des Dispositif ${ }^{5}$ fokussiert Clarke in der Situationsanalyse die Analyse von Relationen (Machtbeziehungen, Bedeutungszuweisung etc.) zwischen einzelnen Elementen (Diskursen, Medien, Personen), nicht die Analyse der Elemente an sich (Clarke et al. 2018).

\subsection{Einbezug Nicht-menschlicher Elemente und Aktanten}

Als nächsten Schritt ergänzt Clarke die GT - und die Soziale Welten/Arenen Theorie - um die poststrukturalistische Beschäftigung mit der Handlungsfähigkeit des Nicht-Menschlichen in Anlehnung an den Pragmatismus, den Interaktionismus und die Science \& Technology Studies (STS), insbesondere die Actor-Network-Theory

5 Verstanden als "the system of relations that can be established between these [Diskurse, Institutionen, Gesetze, Moralvorstellungen etc.; eigene Anm.] elements» (Foucault 1980). 
(ANT), die u.a. von Latour (1987) entwickelt wurde (Clarke et al. 2018). Dabei betont Clarke, dass Objekte im Pragmatismus und Interaktionismus seit jeher berücksichtigt werden würden und in diesen Arbeiten - wie auch bei Foucault und in der Situationsanalyse - die Analyse der Relationen zentral sei. Jedoch seien Objekte dabei nie explizit einbezogen und die Analyse ihrer Handlungsfähigkeit nicht methodologisch ausgearbeitet worden. Auch Böschen et al. (2015) weisen darauf hin, dass sich die meisten Publikationen zur Material Agency - verschiedener Disziplinen und bezogen auf unterschiedliche soziale Phänomene ${ }^{6}$ - auf die Konzeptualisierung, nicht aber auf Möglichkeiten einer methodologischen Annäherung fokussieren würden. Dabei sei es sowohl die zentrale Anforderung als auch die zentrale Schwierigkeit für die empirische Auseinandersetzung mit Material Agency, Wege zu finden, die Verstrickung von materieller und menschlicher Handlungsfähigkeit zu untersuchen.

Kirchhoff (2009) stellt zwei Positionen innerhalb der MaterialAgency-These entgegen: Konstituieren ein materielles Objekt und ein/e menschliche/r AkteurIn gemeinsam eine Aktivität und erfüllen sie dabei die gleichen Funktionen, verstehe der weak view das materielle Objekt und den/die menschliche/n AkteurIn als kausal gekoppeltes System, demzufolge auch das materielle Objekt bei der Analyse der betreffenden Aktivität berücksichtigt werden müsse. Der strong view, dem er u.a. die ANT zuordnet, gehe unter den gleichen Bedingungen einen Schritt weiter und konstatiere, dass es keinen auf ihrer 〈Materialität〉 basierenden prinzipiellen Unterschied zwischen den beiden geben müsse. Kategorien wie «technologisch» und «sozial» werden hierbei als fliessend verstanden, der Fokus liegt auf der Relationalität zwischen den Kategorien, die entsprechende Handlung wird als Ergebnis der Kooperation innerhalb des Netzwerks betrachtet (Latour 1999). Zu nennen ist hier auch die Material Engagement Theory (MET; Malafouris 2013). Der MET zufolge

6 Beispiele für Auseinandersetzungen mit Material Agency lassen sich u.a. bezogen auf Mensch-Maschine-Interaktion (z.B. Fink und Weyer 2014), Tourismus (z.B. Ren 2010), Arbeitsplatz (z.B. Suchman 2007) und Umweltbewegungen (z.B. Lockie 2004) finden. 
übernehmen geschaffene (materielle) Umgebungen «an active role in the structure of agency» (Ransom 2017, 2). Diese explizite Einbindung nicht-menschlicher Elemente und Aktanten, wie sie in den hier erwähnten strong views vertreten werden, stellt nicht nur eine wichtige Passung zwischen Situational Analysis und der Mediatisierungsforschung her, sondern gibt auch wichtige Impulse für die medienpädagogische Forschung und Theoriebildung.

Die zunehmende Verbreitung von softwarebasierten Medien lässt den Unterschied zwischen menschlichen Akteurinnen und Akteuren sowie nicht-menschlichen Aktanten noch kleiner erscheinen. Software kann als durch den menschlichen Akt des Programmierens in (Programm-)Code encodierte Agency verstanden werden. Wenn ein Programm läuft, stellt sich die Frage, wer AkteurIn oder Aktant ist. Die laufende Software? Oder der/die ProgrammiererIn? Und da sich die Software ja in der Hardware materialisiert (als Prozessor-Design, als in Chipdesigns implementierte Algorithmen z.B. zur Video-Komprimierung und -Dekomprimierung) bzw. als Software-Download oder -Update die Praxen der Hardwarenutzung verändern kann (z.B. durch eine neue App auf dem Smartphone oder eine neue Funktion in einem Update) müssen alle technischen Medien in die Analyse aufgenommen werden.

Die Situationsanalyse scheint hier eine geeignete Methode, um alle in der Situation - und speziell für die Interaktion in der Situation (siehe Strübing 2018) - relevanten Elemente («nicht-menschliche) und (menschliche)) als kommunikative Figuration herauszuarbeiten und in ihren Relationen analytisch greifbar zu machen. Unter Einbezug der Diskurs-Theorie nach Foucault werden dabei auch explizit Machtverhältnisse berücksichtigt und analysierbar gemacht.

Analytisch besonders vielversprechend für Fragen der Medienpädagogik scheinen hier u.a. Fragen nach dem damit einhergehenden (Empowerment). Tutorials oder Erklärvideos (siehe Definitionen Wolf 2015) beispielsweise, haben das Potential, Laien-Produzierende 
durch ihre eigene Handlung - ermöglicht durch Medien - zu einer Art Expertinnen- und Experten-Status zu verhelfen bzw. diesen zu kommunizieren, während sich Nutzende durch ihre selbstbestimmte Aneignung der auf diese Weise medial kommunizierten Kompetenzen oder Wissensinhalte potenziell selbst zu Expertinnen und Experten bilden können. Dabei werfen diese mit der Mediatisierung einhergehenden Möglichkeiten der Mediennutzung neue Fragen nach dem veränderten Verhältnis formalen und informellen Lernens und den sich so (potenziell) transformierenden Machtverhältnissen zwischen Expertinnen und Experten (z.B. Lehrpersonen) und Laien (z.B. Schülerinnen und Schüler) auf. Eine weitere Frage ist, wie (bzw. ob) sich die Handlungsfähigkeit von Produzierenden, Medien und Nutzende unterscheidet, wie sie sich wechselseitig beeinflusst, wer also wie zur Handlungsfähigkeit aller anderen Elemente einer Situation beiträgt.

\subsection{Identifikation von impliziten / zum Schweigen gebrach- ten / versteckten Akteurinnen und Akteure}

Im gesamten Forschungsprozess der Situationsanalyse sollte sich der/die Forschende immer wieder die Fragen stellen, was oder wer zusätzlich relevant sein könnte, aber noch nicht genannt wurde, und warum diese/r AkteurIn, dieser nicht-menschliche Aktant, diese Diskursposition keine Erwähnung findet. Clarke (z.B. 2005) nennt diese Akteurinnen, Akteure und Aktanten implicated/silent actors/actants. Dies können zum einen Personen sein, die physisch anwesend sind, aber nicht erhört, sondern ignoriert und/oder übersehen werden; zum anderen Personen, die physisch nicht anwesend sind, aber innerhalb der sozialen Welt diskursiv konstruiert werden. Die zentralen analytischen Fragen dabei sind «Whose constructions of whom/ what exist? Which are taken as the 〈real〉 constructions or the ones that matter most in the situation by the various participants? Which are contested? Whose are ignored? By whom?» (Clarke et al. 2018, 77). Das Konzept der implicated actors and actants sollte dabei als Ausgangslage dienen, um die Machtverhältnisse innerhalb sozialer 
Welten sowie die Situiertheit von weniger machtvollen Akteurinnen, Akteure und Aktanten und die Konsequenzen, die dies für alle Akteurinnen, Akteure und Aktanten innerhalb der Situation hat, zu analysieren.

\subsection{Visualisierung durch Karten}

Alle bisherigen Überlegungen integrierend, entwickelt Clarke drei Typen von Maps, die das Material im Analyse-Prozess zugänglich machen sollen: Situationsmaps, Soziale Welten/Arenen Maps und Positionsmaps. Diese visuelle Darstellung lehnt sich an soziologische Karten der Chicago School of Sociology und damit auch an die Sozialen Welten/Arenen von Strauss an. Diese im nächsten Kapitel zu beschreibenden Karten dienen insbesondere der Unterstützung des Forschungsprozesses, können jedoch auch zur Ergebniskommunikation beitragen.

Die Praxis des Mappings innerhalb der Situationsanalyse wird im Folgenden entlang einer Beispielstudie von Wolf und Wudarski (2017) verdeutlicht.

\section{Maps der kommunikativen Figurationen des in- formellen Lernens}

Das Ziel der hier exemplarisch beschriebenen Studie «Kommunikative Figurationen des informellen Lernens» (Wolf und Wudarski 2017) war die Untersuchung des autodidaktisch-informellen Lernens von Amateurinnen und Amateuren in zwei ausgewählten Domänen. Das Projekt war explizit als eine interdisziplinäre Kombination aus Medien- und Kommunikationsforschung sowie Bildungsforschung konzipiert (sensu Drotner und Erstad 2014). Als Lerndomänen dienten zwei Praxisdomänen DIY (Do It Yourself) / MAKER sowie MOG (Multiplayer-Online-Gaming), welche sich durch überwiegend informelle Lernkulturen und eine hohe Beteiligung von Amateurinnen und Amateuren beschreiben lassen, welche aber unterschiedlich 
enge Bezüge zur Digitalisierung haben (Wolf und Wudarski 2017, 131f.).

Die grundlegende Frage der Studie war, ob und auf welche Weise Amateurinnen und Amateure in einer sich verändernden Medienumgebung neue Zugänge der Selbstexpertisierung wahrnehmen oder schaffen. Der Diskurs zu diesem Thema reicht von euphorischen Beschreibungen einer potentiellen Ermächtigung (Ito et al. 2009; Thomas und Brown 2011; Gee 2013) bis hin zu kulturpessimistischablehnenden Positionen (Keen 2007; Carr 2011; Lanier 2013; Selwyn 2013) und empirischen Studien, welche zumindest Zweifel an der Breite des Phänomens aufkommen lassen (Gibbons 2008; Ragnedda und Muschert 2013; Lane 2009; Friesen und Lowe 2012). Basierend auf dem theoretischen Modell kommunikativer Figurationen sollten mittels einer Kombination von GTM und Situationsanalyse folgende Ziele erreicht werden:

- Figurative Rekonstruktion der Selbstexpertisierung in zwei Domänen;

- Vergleich dieser Figurationen zwischen den Inhaltsdomänen;

- Identifikation von (Diskurs-)Arenen des informellen Lernens;

- Identifikation von Diskurspositionen.

Da sowohl Situationsanalyse als auch GTM keine Vorgaben zur Datensammlung machen, ist zunächst zu klären, welche Datenerhebungsformen sich zur empirischen Erforschung kommunikativer Figurationen des Lernens anbieten. Als mögliche Formen wurden zunächst identifiziert: (1) Interviews mit Lernenden; (2) Teilnehmende Beobachtung in analogen Lernkontexten; (3) Netnographische Analysen von mediatisierten Lernkollektiven; (4) (Online-)Fragebogenerhebungen in abgegrenzten Lernkollektiven und Communities; (5) Lern- und Medientagebücher bzw. automatisiertes Tracking digitaler Nutzungsprozesse; (6) Digital Methods, insbesondere Data Scraping, also das Sammeln von Daten wie z.B. Kommentaren, Likes oder Playlists auf digitalen Online-Medien. 
Zentrale Datenerhebungsmethoden in der hier beschriebenen Studie waren überwiegend Interviews, teilnehmende Beobachtung und Netnographie. Nur so konnten sowohl die analoge, passiv-digitale als auch die aktiv-digitale Nutzung rekonstruiert werden. Interviews sind dabei besonders geeignet, die Breite individueller Kommuikationsrepertoires zu erschliessen (Klein et al. 2016). Die teilnehmende Beobachtung ist für das Erfassen impliziter und nicht-bewusster Lern- und Mediennutzungsprozesse wichtig. So hatten z.B. Lernende in der Domäne DIY-Trendsportarten in Interviews von einer kritischen Einstellung zu sozialen Medien sowie einer reduzierten Nutzung berichtet, in der teilnehmenden Beobachtung in offenen Werkstätten oder Skateparks wurde jedoch offensichtlich, dass insbesondere Filme sowie Informationen über neues «Gear» laufend auf mobilen Geräten angeschaut, geposted, geliked und kommentiert wurden und für das Erlernen neuen Wissens oder als Inspiration für neue Lernprojekte eine zentrale Rolle einnehmen. Netnographische Analysen erkunden die digitalen Spuren der Lernkommunikation mediatisierter Lernkollektive (Wolf und Breiter 2014): Individuen dokumentieren online ihre Problemlöse-Prozesse, machen diese somit für andere findbar und helfen sich dadurch gegenseitig. Über die Online-Analyse dieser Kommunikationspraxen lassen sich insbesondere die öffentlichen und weit verbreiteten Teile kommunikativer Figurationen in Form der Kommunikationsensembles und Akteurskonstellationen nachzeichnen sowie Diskurse analysieren.

Fragebogenerhebungen, Tagebücher und Data Scraping wurden in der hier vorgestellten Studie nicht genutzt, sie dienen insbesondere einer datenmässigen quantitativen Unterfütterung der bereits vorgestellten Erhebungsmethoden, indem z.B. durch eine OnlineErhebung die Häufigkeit der Nutzung einzelner Elemente eines domänenspezifischen Medienensembles erhoben wird. Data Scraping kann allerdings auch als Basis für weitere qualitative Analysen genutzt werden, wenn z.B. Diskussionen in Foren zu Lernthemen für eine qualitative Inhaltsanalyse oder eine kritische Diskursanalyse extrahiert werden. 
Im Folgenden werden die einzelnen Arten von Maps anhand von Ergebnissen der Studie von Wolf und Wudarksi (2017) vorgestellt und veranschaulicht.

\subsection{Situationsmaps/-karten des informellen Lernens}

Clarke benennt ein ganzes Set möglicher Elemente einer Situation. Für diese sollen im Folgenden einige wenige Beispiele aus den Situationsmaps der Lerndomänen DIY_MAKER und MOG (Wolf und Wudarski 2017) gegeben werden:

Individuelle menschliche Elemente / Akteurinnen und Akteure: dies sind zunächst natürlich alle Interviewpartnerinnen und -partner, aber auch deren Kommunikations-, Lernpartnerinnen und -partner, besonders bekannte Personen, wie z.B. «Let's Play»-YouTuberinnen und YouTuber oder Tutorial-Produzierende;

1. Nicht-menschliche Elemente / Aktanten: dies können z.B. TrainingsBots in Spielen sein, Selektionsalgorithmen auf YouTube, Fablabs, Live-Streaming, Spielmechanismen, vielfältige Medienformate und Onlinedienste wie z.B. Twitch oder TeamSpeak;

2. Kollektive menschliche Elemente / Akteurinnen und Akteure: Facebook-Gruppen, Spielefirmen, Clans, Hersteller, Forenmitglieder, Vereinsmitglieder;

3. Implizite / zum Schweigen gebrachte Akteurinnen und Akteure / Aktanten: weibliche Spielerinnen;

4. Diskursive Konstruktionen individueller oder kollektiver menschlicher Akteurinnen und Akteure: süchtige Videospielerinnen und -spieler, Celebrity-Gamerinnen und -Gamer, «Nerds», kreative Makerinnen und -Maker, profitorientierte Computerspiel-Publisher;

5. Diskursive Konstruktionen nicht-menschlicher Aktanten: spielzeitverlängernde Computerspielgestaltung, Jugendschutz;

6. Politische/wirtschaftliche Elemente: In-App-Purchases, Maker-Kodex, Datenschutz;

7. Soziokulturelle / symbolische Elemente: Gender, Hacker-/MakerKultur; 
8. Temporale \& räumliche Elemente: offene Werkstätten, E-SportEvents;

9. Zentrale Streitpunkte und Debatten: Kommerzialisierung, Teilen;

10. Verwandte / zugehörige Diskurse: Gewalt in Spielen; und

11. ggf. andere Elemente.

In der Analyse kommunikativer Figurationen sind die ersten Versionen der Situationsmaps zunächst ein Annähern an die zu betrachtende Figuration. Aus unserer Sicht erscheint die Erstellung von Situationsmaps in jeder GTM-Studie - und insbesondere bei medienpädagogischen Forschungsprojekten - sinnvoll, da sie bei der Strukturierung des Untersuchungsraumes sowie dem Theoretical Sampling wertvolle Dienste leisten, auch wenn man die Situationsmap nur für die Analyse nutzt und weitere Arten von Maps der Situationsanalyse nicht erstellt. Um nun diese Figurationen innerhalb ausgewählter Lerndomänen aus einer situativen Perspektive zu rekonstruieren bzw. zwischen diesen zu vergleichen, bedarf es Multi-Site-Studien, also eines Untersuchungsdesigns, welches eine Datenerhebung an verschiedenen «Orten» (Material) umfasst. Dies ist notwendig, um getrennte Teile einer Figuration zu entdecken. In der Studie von Wolf und Wudarski waren dies neben überwiegend teilnehmenden Beobachtungen und ad-hoc-Interviews in Clan-Häusern/-Wohnungen, E-Sport Events, Fablabs, offene Workshops und Fachgeschäfte sowie Netnographie auf einer Vielzahl von Webseiten und in Apps.

Zum Beispiel konnten Wolf und Wudarski (2017) in der Domäne des Handarbeitens ältere Lernende identifizieren, welche nur analoge Medien wie gedruckte Zeitschriften und Bücher, mündliche Kommunikation mit Familienmitgliedern und Freundinnen in Privathaushalten sowie mit Besitzerinnen und Besitzern bzw. Angestellten in Strickwarenläden bzw. -abteilungen zum Lernen nutzten. Eine andere Gruppe überwiegend junger, ausschliesslich weiblicher Lernerinnen wurde über YouTube auf das Stricken aufmerksam, nutzte dann wachsende digitale Medienrepertoires wie z.B. Pinterest, Blogs und Craftsy, aber keinerlei analoge Medien. Um eine umfassende Beschreibung der Konfiguration zu entwickeln, war es also 
im Sinne eines Theoretical Samplings notwendig, «analoge» Orte zu erkunden, um wichtige Offline-Bereiche einer Lernfiguration zu entdecken, und umgekehrt (siehe auch Positionsmaps weiter unten). Auch fanden Wolf und Wudarski dort Lernende, welche Online-Medien nur passiv nutzten, diese jedoch ausdruckten und in ihre analogen informellen Lerngruppen einführten. In sich anschliessenden, vertiefenden Interviews mit jüngeren Lernenden mit eigenen YouTube-Channels oder Blogs konnte herausgearbeitet werden, dass diese teilweise aktiv das Wissen z.B. ihrer älteren «Nonliner»-Verwandten in analogen Kommunikationskontexten erkundeten oder alte Bücher aus dem Antiquariat suchten, um dieses Wissen dann wieder auf ihren YouTube-Kanälen oder Blogs in Form von Tutorials zu teilen.

In dem Projekt wurden aus den Situationsmaps verschiedene «Schnitte» angefertigt, welche insbesondere zur Analyse der Medienensembles dienten. Situationsmaps sind gute Werkzeuge, um die Situationen $\mathrm{zu}$ «befragen», allerdings eignen sie sich bei hinreichender Komplexität weniger zur Darstellung in ihrer Ganzheit. Bezogen auf eine spezifische Fragestellung, wie z.B. die Konstituierung von domänenspezifischen Medienensembles in ihrer Widersprüchlichkeit, Heterogenität oder Fragmentierung, sind sie sehr hilfreich.

Ausgehend von kommunikationsbezogenen Situationsmaps der einzelnen Interviewpartnerinnen und -partner verdichtet man schrittweise zu einem hochaggregierten Schnitt durch die kollektive Situation. Abbildung 1 zeigt als Beispiel einen Vergleich der hochaggregierten Medienensembles der beiden Lerndomänen DIY_MAKER und MOG. Durch die Erstellung und Analyse der Situationsmaps umfassen diese nicht nur «klassische» oder «digitale» Medien wie Bücher, Facebook oder Webseiten. Vielmehr werden weitere nichtmenschliche Elemente / Aktanten sowie temporale und räumliche Elemente als Teil des Medienensembles verstanden. So konstituieren im DIY_MAKER-Medienensemble Ebay und Online-Shops, aber auch Kataloge, Pinterest, Thingiverse sowie Repositorien, Werkstätten 
und Messen kollektive Lernräume. Eine Werkstätte im Kontext des informellen Lernens ist somit nicht nur ein Raum zur direkten Kommunikation, sondern ein Medium zur reziproken oder produzierten Medienkommunikation, in dem z.B. die dort angeordneten und zur Verfügung gestellten Geräte als Aktanten für die informellen Lernprozesse dienen, man face-to-face voneinander lernt, Tutorials produziert oder durch die sich in Produktion befindlichen Projekte der anderen Akteurinnen und Akteure zu eigenen Lernprozessen angeregt wird.

Weitere «Dimensionen» einer Situationsmap des informellen Lernens sind die Akteurinnen und Akteure / Aktanten sowie deren kommunikativen Praxen. Zu der obigen Darstellung könnte man also die Medienaktivität hinzufügen und als einen ersten einfachen analytischen Schritt zwischen konsumptiver und produzierender Nutzung von Medien (aktiv/passiv) unterscheiden, also z.B. Lesen vs. Schreiben von Twitter-Beiträgen oder Lesen vs. Bearbeiten von Wiki-Artikeln. Innerhalb des hier betrachteten Kommunikationsensembles waren diese Aktivitätstypen weitaus differenzierter. So kann z.B. das Schreiben eines Blogs das einfache Posten von Beiträgen sein, es kann aber auch intensive Interaktion mit anderen Bloggerinnen, Blogger und Lesenden über Kommentare, Backlinks oder Blogparaden bedeuten. So können auch bei gleicher Zusammensetzung eines Medienensembles unterschiedliche Nutzendengruppen anhand ihrer verschiedenen Nutzungsaktivitäten der gleichen $\mathrm{Me}-$ dien identifiziert werden. 


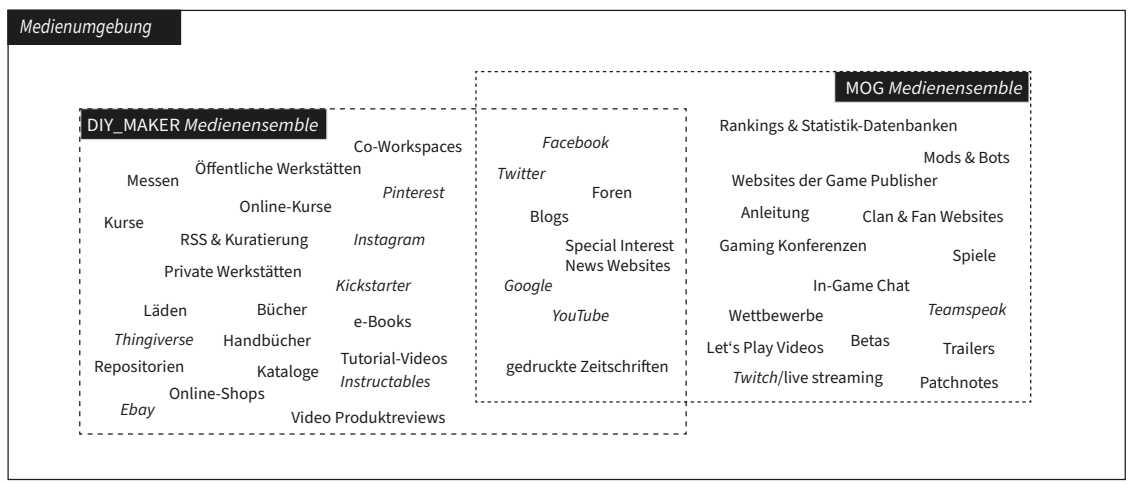

Abb. 1.: Hochaggregierte Map der Medienensembles der beiden Lerndomänen DIY_MAKER und Multiuser Online Gaming (MOG). Quelle: Wolf und Wudarski 2017; eigene Übersetzung.

Die Situationsmap scheint insgesamt eine geeignete Methode zu sein, um die Zusammenhänge zwischen allen hier in Einzelschritten erhobenen Elementen zu integrieren und die Daten so für die Analyse greifbarer zu machen. Speziell die Verstrickung von Menschen und Kommunikationsmedien in ihren Handlungen könnte mithilfe einer Situationsmap abgebildet und so analytisch zugänglicher gemacht werden.

\subsection{2 Soziale Welten/Arenen Maps des informellen Lernens}

Soziale Welten/Arenen Maps umfassen alle Diskurse, Identitäten, geteilte Werte, Handlungsorte, Beziehungen etc., die im Verlauf eines Projektes herausgearbeitet werden. Clarke et al. $(2018,148)$ definieren Soziale Welten als

«groupings of varying sizes, each of which has 〈a life of its own〉 that is distinctively collective [...]. Participants in social worlds generate shared perspectives that form the basis for both individual and collective identities.»

Dabei gibt es nicht nur Differenzen zwischen sich unterscheidenden sozialen Welten (《interworld differences»), sondern auch innerhalb einer sozialen Welt (《intraworld differences») bezüglich einzelner Perspektiven und Eigenschaften. Diese Unterschiede können zu 
Aufspaltungen führen, die Bucher (1962) als Segmente oder Subwelten bezeichnet. Arenen bestehen aus vielfältigen sozialen Welten. In ihnen werden vielfältige Probleme diskutiert und Konflikte ausgetragen (Strauss 1978). Da in Arenen viele unterschiedliche soziale Welten aufeinandertreffen, können Arenen, die über einen langen Zeitraum bestehen, als 〈Orte〉 vielfältiger und komplexer Diskurse verstanden werden. Um eine spezifische soziale Welt verstehen zu können, ist es unerlässlich I) die Arenen zu verstehen, in die die soziale Welt eingebunden ist; 2) die anderen sozialen Welten zu verstehen, auf die sie in den Arenen trifft, sowie die Diskurse, die dort verhandelt werden (Clarke et al. 2018).

Soziale Welten/Arenen Maps werden auf Basis des Datenmaterials des gesamten Projektes erstellt. Wird während des Prozesses bereits mit der Erstellung dieser Maps begonnen, sollten diese im weiteren Verlauf immer wieder überarbeitet und die ursprünglichen Maps zur Nachvollziehbarzeit des Auswertungsprozesses aufbewahrt und Memos verfasst werden. In ihrer Untersuchung sozialer Arenen fokussierten Wolf und Wudarski (2017) die Akteurinnen und Akteure in den kommunikativen Figurationen des informellen Lernens und deren Diskurse: Lernende als individuelle Akteurinnen und Akteure, Gruppen von gemeinsam problemlösenden Lernenden als kollektive Akteurinnen und Akteure, kommerzielle Akteurinnen und Akteure wie Verlage und Trainingsanbietende.

In der MOG-Akteurskonstellation werden die meisten (relevanten) Spiele von kommerziellen Firmen produziert. MOG stellen einen grossen Markt für Computerspiele dar, erfordern aber neben der kostspieligen Entwicklung auch weitere ressourcenintensive Dienste wie Server. Selbst aus der Gemeinschaft der Spielenden entwickelte "Community-Mods» (nicht kommerzielle Spielerweiterungen) wurden kommerzialisiert, so wie das populäre MOBA Defense of the Ancients (Dota) oder das FPS Counter Strike. Durch ihre wettbewerbsorientierte Struktur sind MOGs besonders für die 
Umsetzung als E-Sport mit hohen Preisgeldern geeignet. Zusammen mit einer professionellen Presseberichtserstattung hat sich in der Akteurskonstellation von MOG dadurch eine starke Diskursarena der Kommerzialisierung entwickelt, in der mittlerweile auch spielefremde Marken als Sponsoren agieren. Beispielhafte Diskurse sind z.B. der Streit um neue Geschäftsmodelle der Spieleindustrie in Form von F2P (Free 2 Play), DLC (Downloadable Content) und IAP (In-App-Purchases) und ihre Auswirkungen auf die Gestaltung von Spielen. Es finden sich verschiedene Positionen von Kritik aus dem Journalismus (Spielepresse) bezüglich der Zerstörung des Spielspasses bis hin zu Optimierungsdiskussionen der Spieleentwickler. Aktuell wurde in dieser Diskursarena sogar der Gesetzgeber aktiv, als das Verbot von sogenannten «Loot-Boxen» diskutiert und in einigen Ländern rechtlich umgesetzt wurde.

Eine weitere Diskursarena ist das Meta-Game. In dieser werden alle Spielmechanismen und deren Änderung diskutiert. SpieleEntwickelnde befinden sich im Zentrum sowohl der Kommerzialisierungs- und der Meta-Game-Arena. Ihre Aufgabe ist es, die Spiele für die Spielenden (Spass) und die Produktionsfirmen (Gewinn) möglichst attraktiv zu gestalten. Im Zentrum der Diskursarena des Lernens befinden sich ernsthafte bzw. wettbewerbsorientierte Amateurspielerinnen und -spieler, welche untereinander ihr Wissen austauschen, und kommerziell orientierte Twitch-Streamerinnen, -streamer und Let's Play-Produzierende auf YouTube. Gelegenheitsspielerinnen und -spieler (casual player) und nicht wettbewerbsorientiere Hobbyspielerinnen und -spieler beteiligen sich weniger an lernrelevanten Meta-Game-Diskursen. Mit einem höheren Wissen über das Meta-Game werden auch Spielende einflussreicher auf den Meta-Game-Diskurs und können dadurch die Entwicklung von zukünftigen Patches und Spielen beeinflussen. Hier finden sich dann auch indirekt mit dem Spielen berührte Akteurinnen und Akteure, wie z.B. Eltern, Partnerinnen und -partner bzw. ein jeweils fehlendes soziales Umfeld, welche Teil des Meta-Game-Diskurses zu Sucht/ Exzessivem Spielen sind. In Abbildung 2 sind die in drei relevanten 
Diskursarenen, Kommerzialisierung, Meta-Game und Lernen, beteiligten Akteurinnen und Akteure angeordnet. Horizontal ergeben sich verschiedene Machtpositionen von Spielen über Analysieren hin zu Beeinflussen und Definieren. Dabei ist zu berücksichtigen, dass sich die Akteurinnen und Akteure über Lernen von einfachen Casual Players zu einflussreichen Let's Player-innen entwickeln können, und dass die einzelne Machtposition von Millionen von Spielenden über geteilte ablehnende Positionen z.B. zum DLC/IAP in der Summe zu neuen Machtbalancen führen können (z.B. durch Kaufboykott bzw. niedrigere Umsatzzahlen), welche dann zu Korrekturen auf Seiten der Game Publisher und damit der Spieleentwickler führen.

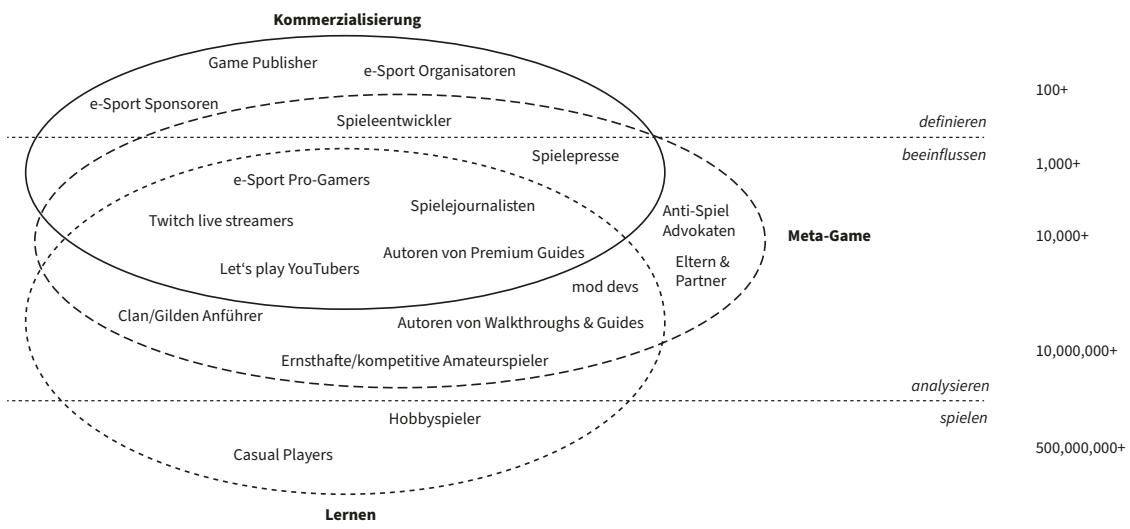

Abb. 2.: Akteurinnen und Akteure des Lernens und relevante Diskursarenen beim Multiplayer Online Gaming. Quelle: Wolf und Wudarski 2017; eigene Übersetzung.

Im Vergleich dazu formen sich bei DIY_MAKER (siehe Abbildung 3) deutlich egalitärere Akteurskonstellationen heraus, da es weder Ranglisten noch andere wettbewerbsorientierte Elemente gibt. Zum Beispiel erklärte eine vegane Foodbloggerin, dass sie das meiste von den Kommentaren und Ideen ihrer Lesenden lerne, die oft ihre Rezepte veränderten, erweiterten und ihr neues Wissen über Kommentare teilten. Zwar fand sich auch in dieser Lerndomäne eine Soziale Arena der Kommerzialisierung, die aber viel kleiner ist und eine geringere Machtposition aufweist als bei MOG. Während die Computerspielebranche riesig ist (so machte z.B. das Spiele-Unternehmen 
Electronic Arts laut eigenen Angaben 4,85 Mrd. \$ Umsatz in 2017), sind wichtige MAKER-Projekte wie z.B. die Physical-ComputingPlattform Arduino Open Source Projekte, welche nicht primär auf eine kommerzielle Umsatz- oder Gewinnmaximierung zielen. Da es sehr viele unterschiedliche Interessen und Projekte in DIY_MAKER gibt, sind darüber hinaus die involvierten Firmen kleiner und wenig bestimmend. Die zentrale Diskursarena in der DIY_MAKERAkteurskonstellation ist «Teilen und Gemeinschaft», da die meisten Innovationen und Lernressourcen kollaborativ erstellt werden, so dass selbst die kommerzielle DIY_MAKER-Presse an diesen Aktivitäten des Teilens teilnimmt.

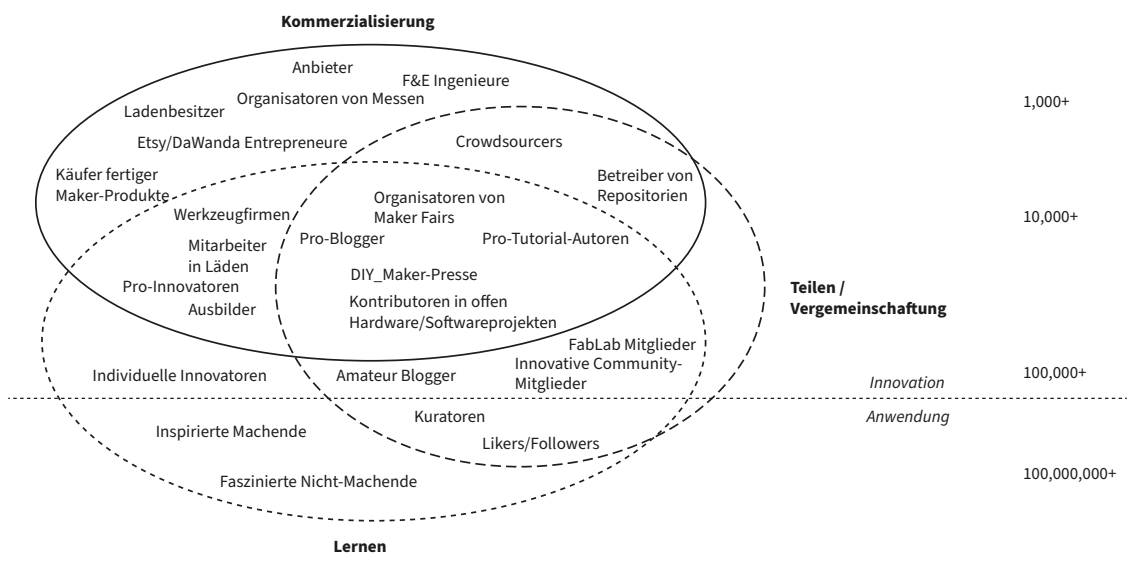

Abb. 3.: Akteurskonstellation in der Lerndomäne DIY_MAKER. Quelle: Wolf und Wudarski 2017; eigene Übersetzung.

\subsection{Positionsmaps des informellen Lernens}

Positionsmaps widmen sich vollständig der Visualisierung von verschiedenen Diskurspositionen - auch der nicht genannten - innerhalb der relevanten Diskurse und der Herausarbeitung von Ähnlichkeiten und Unterschieden. Ziel ist es dabei, die Diskurse zu ihren eigenen Bedingungen und in ihren eigenen Worten abzubilden. Positionsmaps tragen so dazu bei, die Heterogenität und Konflikte innerhalb eines Diskurses deutlich zu machen und die gegensätzlichen Perspektiven der Beteiligten zu systematisieren. Als hilfreiche 
Fragen für die Analyse von Diskursen nennen Clarke et al. (2018) u.a. Warum sprechen Menschen darüber? Warum scheint es für sie so wichtig zu sein? und Wer innerhalb meines Datenmaterials nimmt eine gegensätzliche Position ein? Warum? Wie kann das Verhältnis zwischen den Positionen beschrieben werden? Insgesamt liegt der Fokus bei Positions- sowie Soziale Welten/Arenen Maps - in Abgrenzung zu einer positivistischen Ausrichtung der Grounded Theory nach Strauss und Glaser - auf der Rekonstruktion und Herausarbeitung von Komplexität, insbesondere Variationen und Differenzen (Clarke 2011b).

Während also Situationsmaps vor allem der Darstellung der Komplexität dienen, fokussieren Positionsmaps eher auf die poignante Gegenüberstellung, aber auch die Identifizierung möglicher von den Forschenden selbst übersehener bzw. nicht entdeckter Positionen, was im Sinne des theoretischen Sampling der GTM zu weiteren Suchbewegungen im untersuchten Forschungsfeld anregen sollte. Nachfolgend ist in Abbildung 4 ein einfaches Beispiel für die Positionsmaps der Expertisierungsmotivation in MultiplayerOnline-Games dargestellt. Die Expertisierungsmotivation in MOG ist aus vielerlei Perspektiven interessant. So ist es für Spieleproduzierende wichtig, ein Spiel entwickeln zu lassen, welches herausfordernd, aber erlernbar ist. Pädagoginnen und Pädagogen beobachten häufig fasziniert die Energie, die von ihren Schülerinnen und Schüler in das Erlernen eines Spieles gesteckt wird und fragen sich, was sie daraus für ihren Unterricht lernen können; für «Serious Games》Entwickelnde ist es das zentrale Ziel ihrer Lernspiele.

Aus der Social Arena Map wurden dazu zwei dominante Diskursarenen, Wettbewerb und Kommerzialisierung/Kosten, herausgelöst und auf der Positionsmap vier Positionen auf Basis des Datenmaterials identifiziert. Die hier dargestellte Positionsmap in Abbildung 4 hätte wahrscheinlich auch aus einer qualitativen typisierenden Inhaltsanalyse entwickelt werden können - so nah sind die Positionen an typischen Interviewpositionen - vorausgesetzt, die Analysedimensionen wären vorgegeben gewesen. 


\begin{tabular}{|l|l|l|}
\hline+ & $\begin{array}{l}\text { Besser werden um ein/e } \\
\text { stärkere/r Spieler/in zu sein }\end{array}$ & Trainieren, um zu gewinnen \\
\hline Wettbewerb & Mehr vom Spiel erleben & Viel Spielen ohne zu bezahlen \\
\hline- & $-\quad$ Kommerzialisierung / Kosten + \\
\hline
\end{tabular}

Tab. 1.: Positionsmap «Diskursive Positionen der Expertisierungsmotivation in MOG». Quelle: eigene Darstellung.

Die Positionsmap in Abbildung 5 dagegen zeigt für DIY_MAKER durch die explizite Aufnahme des Kommerzialisierungs-Diskurses Positionen, die erst im Laufe der Situationsanalyse durch Theoretical Sampling identifiziert wurden. So erscheint Teilen und Kommerzialisierung zunächst widersprüchlich zu sein. Durch die gezielte Erweiterung der Analyse konnten allmählich mehrere Positionen identifiziert werden. So dient z.B. das offene Repository «Thingiverse» mit 3D-Druck-Konstruktionen der Druckerfirma MakerBot zunächst einmal der Steigerung des Nutzwertes von 3D-Druckern (also dem Produkt der Firma MakerBot, obwohl die Druck-Konstruktionen auch mit anderen 3D-Druckern der Konkurrenz genutzt werden können). In beiden Maps zeigen sich die Vielfältigkeit und auch mögliche Widersprüche in den beiden Lerndomänen, was genau die Zielsetzung einer Situationsanalyse ist.

\begin{tabular}{|l|l|l|}
\hline+ & $\begin{array}{l}\text { Zu einer besseren Gesell- } \\
\text { schaft beitragen }\end{array}$ & $\begin{array}{l}\text { Customer Education \& Empo- } \\
\text { werment zur Marktentwick- } \\
\text { lung }\end{array}$ \\
\hline $\begin{array}{l}\text { Teilen / Verge- } \\
\text { meinschaftung }\end{array}$ & $\begin{array}{l}\text { Gemeinsam Projekte bewäl- } \\
\text { tigen, die man einzeln nicht } \\
\text { schafft }\end{array}$ & $\begin{array}{l}\text { Crowdsourcing erfolgreich } \\
\text { umsetzen }\end{array}$ \\
\hline- & $\begin{array}{l}\text { Dinge haben, die man sich } \\
\text { sonst nicht leisten kann oder } \\
\text { die es so nicht gibt }\end{array}$ & Eigene Ideen vermarkten \\
\hline & \multicolumn{2}{|c|}{- Kommerzialisierung / Kosten + +} \\
\hline
\end{tabular}

Tab. 2.: Positionsmap «Diskursive Positionen der Expertisierungsmotivation in DIY/MAKER». Quelle: eigene Darstellung. 


\section{Zusammenfassung und Ausblick}

In diesem Beitrag wurden zunächst die Konsequenzen der kommunikationswissenschaftlichen Mediatisierungstheorie für die medienpädagogische Theorieentwicklung hinterfragt. Das besondere Potenzial dieses Ansatzes liegt in der Hervorhebung der Betrachtung von Medien als Systemelementen auf einer Mesoebene, welche nicht mehr aus der kommunikativen Konstruktion der Gesellschaft - und damit auch der Bildung - analytisch herausgelöst werden können. Ein Denken und Forschen über Bildung ohne Medien scheint vor diesem Hintergrund nicht mehr sinnvoll.

Herausgearbeitet werden konnte ebenso, dass nach vielen sozialwissenschaftlichen «Turns» die praxeologischen, situativen und sozialkonstruktivistischen Ansätze der Mediatisierungstheorie eine gute Passung haben zu den Erkenntnis- und Modellierungsinteressen sowohl der subjekt- und handlungsbezogenen als auch der kritischen und bildungsorientierten Ansätze der Medienpädagogik. Als eine paradigmatische Theorieentwicklung wurde das Konzept der kommunikativen Figurationen der Bremer und Hamburger Forschungsgruppe "Communicative Figurations» (Hepp und CoFi Research Network 2017) als Form einer nicht-medienzentrierten Analyse kommunikativer Prozesse unter besonderer Berücksichtigung der Medien vorgestellt. Wie diese Position aus der Medienpädagogik aufzugreifen ist, beschreibt der Beitrag anhand eines Forschungsprojektes zum informellen Lernen (Wolf und Wudarski 2017). Dabei wird die forschungsmethodologische Frage in den Vordergrund gerückt. Anknüpfend an die GTM wird der Ansatz der Situationsanalyse nach Adele Clarke beschrieben, welcher eine entscheidende Erweiterung der GTM vornimmt, die eine hohe Passung zum Erkenntnisinteresse und theoretischen Bezugsrahmen unseres Forschungsinteresses aufweist. Basierend auf dem Ziel, die Komplexität und Widersprüchlichkeit von Situationen herausarbeiten zu wollen, werden in der Situationsanalyse Diskurse und nicht-menschliche Elemente und Aktanten in die Analyse einbezogen. Dieser auch für die Kommunikationswissenschaft neue Ansatz integriert also Fragen nach Human/Material Agency und Dis-/Empowerment in die 
Forschungsmethodik und sucht explizit nach impliziten oder versteckten Akteurinnen und Akteuren. Hierfür werden Visualisierungen in Form von Maps/Karten zur Darstellung der qualitativen Auswertungsmethoden vorgeschlagen, die die Diskussion von Ergebnissen erleichtern können.

In der Darstellung der Ergebnisse konnte innerhalb dieses Beitrages nur eine Annäherung an die Vielschichtigkeit der Methode geleistet werden, um erste Potentiale der Methodik zu verdeutlichen. Insbesondere halfen die Situationsmaps beim Analysieren des Datenmaterials sowie beim Theoretical Sampling in einer komplexen Multi-Site-Studie. Die Integration von Theorien zur Material Agency und zum Material Engagement erweitert die bisherigen methodischen Ansätze der kommunikationswissenschaftlichen qualitativen Netzwerkanalyse, um die kommunikativen Konstruktionsprozesse der beteiligten Akteurinnen, Akteure und Aktanten systematischer zu analysieren. Schliesslich führt die Diskursanalyse der Sozialen Arenen sowie der Positionsmaps zu einer in der Medienpädagogik äusserst wichtigen kritischen Auseinandersetzung mit den Aushandlungsprozessen zwischen Menschen, Kommunikationspraxen, Bildung und Medien. Insofern bietet die Situationsanalyse sowohl für kommunikations- und medienwissenschaftliche als auch für medienpädagogische Forschungsprozesse, am besten jedoch für deren Kombination, eine äusserst interessante methodische Plattform.

\section{Literatur}

Anderson, Terry, und Julie Shattuck. 2012. «Design-Based Research: A Decade of Progress in Education Research?» Educational Researcher 41 (1): 16-25. https://doi.org/10.3102/0013189X11428813.

Baacke, Dieter. 1973. Kommunikation und Kompetenz: Grundlegung einer Didaktik der Kommunikation und ihrer Medien. München: Juventa.

Baacke, Dieter. 1999. "Medienkompetenz als zentrales Operationsfeld von Projekten.» In Handbuch Medien: Medienkompetenz. Modelle und Projekte, herausgegeben von Dieter Baacke, Susanne Kornblum, Jürgen Lauffer, Lothar Mikos, und Günter A. Thiele, 31-35. Bonn: Bundeszentrale fuer politische Bildung. 
Berger, Peter L., und Thomas Luckmann. 1967. The social construction of reality: a treatise in the sociology of knowledge. London [u.a.]: Penguin.

Blumer, Herbert. 1969. Symbolic Interactionism: Perspective and Method. Englewood Cliffs, NJ: Prentice-Hall.

Blumer, Herbert. 1973. «Der methodologische Standort des Symbolischen Interaktionismus». In Alltagswissen, Interaktion und gesellschaftliche Wirklichkeit, herausgegeben von Arbeitsgruppe Bielefelder Soziologen, 80-101. Reinbek: Rowohlt.

Blumler, Jay G., und Elihu Katz. 1974. The Uses of Mass Communications: Current Perspectives on Gratifications Research. London et al.: Sage.

Bonfadelli, Heinz, und Thomas N. Friemel. 2014. Medienwirkungsforschung: Grundlagen und theoretische Perspektiven. 5. Aufl. Stuttgart: UTB.

Bongaerts, Gregor. 2007. «Soziale Praxis und Verhalten: Überlegungen zum Practice Turn». Zeitschrift für Soziologie 36 (4): 246-260.

Böschen, Stefan, Jochen Glaser, Martin Meister, und Cornelius Schubert. 2015. «Introduction: Material Agency as a Challenge to Empirical Research». Nature and Culture 10 (3): 257-68.

Bourdieu, Pierre. 1992. The Logic of Practice. Cambridge: Polity.

Bourdieu, Pierre. 1993. The Field of Cultural Production: Essays on Art and Literature. Cambridge: Polity.

Bruns, Axel. 2008. Blogs, Wikipedia, Second Life, and Beyond: From Production to Produsage. New York: Peter Lang.

Bruns, Axel, und Jan-Hinrik Schmidt. 2011. «Produsage: A closer look at continuing developments». The New Review of Hypermedia and Multimedia 17 (1): 3-7.

Bucher, Rue. 1962. «Pathology: A Study of Social Movements within a Profession». Social Problems 10 (1): 40-51. https://doi.org/10.2307/799406.

Butler, Judith. 2001. Psyche der Macht: Das Subjekt der Unterwerfung. Frankfurt am Main: Suhrkamp.

Carr, Nicholas G. 2001. The Shallows: What the Internet is Doing to our Brains. New York: W. W. Norton.

Certeau, Michel de. 2002. The Practice of Everyday Life. Berkeley: California University Press.

Clarke, Adele E. 2005. Situational Analysis: Grounded Theory after the Postmodern Turn. London et al.: Sage.

Clarke, Adele E. 2011a. "Social Worlds». In The Concise Encyclopedia of Sociology, herausgegeben von George Ritzer und J. Michael Ryan, 384-85. Malden: Wiley-Blackwell. 
Clarke, Adele E. 2011b. «Von der Grounded-Theory-Methodologie zur Situationsanalyse». In Grounded Theory Reader, herausgegeben von Günter Mey und Katja Mruck, 2. Aufl., 207-29. Wiesbaden: VS Verlag für Sozialwissenschaften. https:/doi.org/10.1007/978-3-531-933184_10.

Clarke, Adele E., Carrie Friese, und Rachel S. Washburn. 2018. Situational Analysis: Grounded Theory After the Interpretive Turn. 2. Aufl. Thousand Oaks, CA et al.: Sage.

Corbin, Juliet, und Anselm Strauss. 2015. Basics of Qualitative Research: Grounded Theory Procedures and Techniques. Thousand Oaks, CA: Sage.

Couldry, Nick. 2004. «Theorising Media as Practice». Social Semiotics 14 (2): $115-32$.

Couldry, Nick, und Andreas Hepp. 2016. The mediated construction of reality. Cambridge, UK et al.: John Wiley \& Sons.

Drotner, Kirsten, und Ola Erstad. 2014. «Inclusive Media Literacies: Interlacing Media Studies and Education Studies». International Journal of Learning and Media 4 (2): 19-34.

Elias, Norbert. 1978. What Is Sociology? London: Hutchinson.

Fink, Robin D., und Johannes Weyer. 2014. «Interaction of Human Actors and Non-Human Agents. A Sociological Simulation Model of Hybrid Systems». Science, Technology \& Innovation Studies 10 (1): 47-64.

Fornäs, Johan. 2000. «The Crucial in Between: The Centrality of Mediation in Cultural Studies». European Journal of Cultural Studies 3 (1): 45-65.

Foucault, Michel. 1980. Power/Knowledge: Selected Interviews and Other Writings 1972-1977. Herausgegeben von Colin Gordon. New York: Pantheon.

Foucault, Michel. 1988. «Technologies of the Self». In Technologies of the Self: A Seminar with Michel Foucault, 16-49. Amherst: University of Massachusetts Press.

Friesen, Norm, und Shannon Lowe. 2012. «The Questionable Promise of Social Media for Education: Connective Learning and the Commercial Imperative». Journal of Computer Assisted Learning 28 (3): 183-94.

Gee, James P. 2013. Good Video Games and Good Learning: Collected Essays on Video Games, Learning, and Literacy. 2. Aufl. 27. New York: Peter Lang.

Gibbons, Michael C., Hrsg. 2008. EHealth Solutions for Healthcare Disparities. New York: Springer-Verlag. https://doi.org/10.1007/978-0-38772815-5. 
Giddens, Anthony. 1984. The Constitution of Society: Outline of the Theory of Structuration. Cambridge et al.: Polity.

Glaser, Barney G., und Anselm L. Strauss. 1967. The Discovery of Grounded Theory: Strategies for Qualitative Research. New York: Aldine.

Hall, Stuart. 1973. «Encoding and Decoding in Televsion Discourse. Centre for Contemporary Cultural Studies». Occasional Papers 7: 1-12.

Hasebrink, Uwe. 2003. «Nutzungsforschung». In Öffentliche Kommunikation: Handbuch Kommunikations- und Medienwissenschaft, herausgegeben von Günter Bentele, Hans-Bernd Brosius, und Otfried Jarren, 101-27. Wiesbaden: VS Verlag für Sozialwissenschaften. https://doi.org/10.1007/978-3-322-80383-2_6.

Hasebrink, Uwe. 2014. «Die kommunikative Figuration von Familien: Medien, Kommunikation und Informationstechnologie im Familienalltag». In Die Zukunft der Familie: Anforderungen an Familienpolitik und Familienwissenschaft, herausgegeben von Marina Rupp, Olaf Kapella, und Norbert F. Schneider, 225-40. Opladen et al.: Verlag Barbara Budrich.

Hasebrink, Uwe, und Hanna Domeyer. 2012. «Media Repertoires as Patterns of Behaviour and as Meaningful Practices: A Multimethod Approach to Media Use in Converging Media Environments». Participations: Journal of Audience \& Reception Studies 9 (2): 757-83.

Hasebrink, Uwe, und Jutta Popp. 2006. «Media Repertoires as a Result of Selective Media Use. A Conceptual Approach to the Analysis of Patterns of Exposure». Communications 31 (3): 369-387.

Hepp, Andreas. 2005. «Kommunikative Aneignung». In Qualitative $\mathrm{Me}$ dienforschung: Ein Handbuch, herausgegeben von Lothar Mikos und Claudia Wegener, 67-79. Konstanz: UTB (UVK).

Hepp, Andreas, Matthias Berg, und Cindy Roitsch. 2012. «Die Mediatisierung subjektiver Vergemeinschaftungshorizonte: Zur kommunikativen Vernetzung und medienvermittelten Gemeinschaftsbildung junger Menschen». In Mediatisierte Welten: Forschungsfelder und Beschreibungsansätze, herausgegeben von Friedrich Krotz und Andreas Hepp, 227-56. Wiesbaden: VS Verlag für Sozialwissenschaften. https://doi.org/10.1007/978-3-531-94332-9_9.

Hepp, Andreas, und CoFi (Communicative Figurations) Research Network. 2017. «Transforming Communications. Media-Related Changes in Times of Deep Mediatization». Working Paper No. 16. Zugriff 12.12.2018. http://www.zemki.uni-bremen.de/fileadmin/redak_zemki/ dateien/Kofi-Arbeitspapiere/CoFi_EWP_Hepp-Research-Network. pdf. 
Hepp, Andreas, und Uwe Hasebrink. 2017. «How to Research Cross-Media Practices? Investigating Media Repertoires and Media Ensembles». Convergence: The International Journal of Research into New Media Technologies 23 (4): 362-77.

Hjarvard, Stig. 2013. he Mediatization of Culture and Society. London: Routledge.

Hugger, Kai-Uwe. 2012. «Bildung im gegenwärtigen Mediatisierungsprozess». Kulturelle Bildung Online. 2012. Zugriff 12.12.2018. https:// www.kubi-online.de/artikel/bildung-gegenwaertigen-mediatisierungsprozess.

Ito, Mizuko, Sonja Baumer, Matteo Bittanti, danah boyd, Rachel Cody, Becky Herr-Stephenson, Heather A. Horst, u. a. 2009. Hanging Out, Messing Around, and Geeking Out: Kids Living and Learning with New Media. Cambridge MA: MIT University Press Group.

Jörissen, Benjamin. 2015. «Digitale Medien und digitale Netzwerke: Herausforderungen für die Kulturelle Kinder- und Jugendbildung». In Nürnberger Forum der Kinder- und Jugendarbeit 2015, herausgegeben von Bernd Kammerer, 101-19. Nürnberg: emwe-Verlag.

Jörissen, Benjamin. 2017. «Subjektivation und 〈ästhetische Freiheit〉 in der post-digitalen Kultur». Kulturelle Bildung Online. 2017. Zugriff 12.12.2018. https://www.kubi-online.de/artikel/subjektivation-aesthetische-freiheit-post-digitalen-kultur.

Keen, Andrew. 2007. The Cult of the Amateur: How Blogs, MYSpace, YouTube, and the Rest of Today's User-Generated Media Are Destroying Our Economy, Our Culture, and Our Values. New York: Doubleday.

Keppler, Angela. 2005. «Medien und soziale Wirklichkeit». In Mediensoziologie: Grundfragen und Forschungsfelder, herausgegeben von Michael Jäckel, 91-106. Wiesbaden: VS Verlag für Sozialwissenschaften. https://doi.org/10.1007/978-3-322-80675-8_7.

Kirchhoff, Michael. 2009. «Material Agency: A Theoretical Framework for Ascribing Agency to Material Culture». Techne: Research in Philosophy and Technology 13 (3): 206-20.

Klein, Juliane, Uwe Schimank, und Michael Walter. 2016. «How to Interview about Media Repertoires as Tacit Components of Practices - Problems and Empirical Experiences». Communicative Figurations Working Paper, Nr. 12. Zugriff 12.12.2018. https://elib.suub.uni-bremen. de/edocs/00105155-1.pdf.

Knoblauch, Hubert. 2013. «Communicative Constructivism and Mediatization». Communication Theory 23 (3): 297-315. 
Koppel, Ilka. 2017. Entwicklung einer Online-Diagnostik für die Alphabetisierung: Eine Design-Based Research-Studie. Wiesbaden: VS Verlag für Sozialwissenschaften. https://doi.org/10.1007/978-3-658-15769-2.

Krajina, Zlatan, Shaun Moores, und David Morley. 2014. «Non-MediaCentric Media Studies: A Cross-Generational Conversation». European Journal of Cultural Studies 17 (6): 682-700.

Krotz, Friedrich. 2007. Mediatisierung: Fallstudien zum Wandel von Kommunikation. VS Verlag für Sozialwissenschaften. https://doi. org/10.1007/978-3-531-90414-6.

Lane, Andy. 2009. "The Impact of Openness on Bridging Educational Digital Divides». The International Review of Research in Open and Distributed Learning 10 (5).

Lanier, Jaron. 2013. Who Owns the Future? New York: Simon \& Schuster.

Latour, Bruno. 1987. Science in Action: How to Follow Scientists and Engineers Through Society. Cambridge MA: Harvard University Press.

Latour, Bruno. 1999. Pandora's Hope: Essays on the Reality of Science Studies. Cambridge MA: Harvard University Press.

Lockie, Stewart. 2004. "Collective Agency, Non-Human Causality and Environmental Social MovementsA Case Study of the Australian 〈Landcare Movement〉». Journal of Sociology 40: 41-57.

Luckmann, Benita. 1970. «The Small Life-Worlds of Modern Man». Social Research 37 (4): 580-96.

Luhmann, Niklas. 2012. Theory of Society. Stanford: Stanford University Press.

Lundby, Knut. 2014. «Mediatization of Communication». In Mediatization of Communication, herausgegeben von Knut Lundby, 3-35. Berlin, Boston: De Gruyter Mouton.

Malafouris, Lambros. 2013. How things shape the mind: A theory of material engagement. Cambridge: MIT Press.

Meyen, Michael. 2009. «Medialisierung». Medien \& Kommunikationswissenschaft 57 (1): 23-38. https://doi.org/10.5771/1615-634x-2009-1-23.

Moores, Shaun. 2012. Media, Place and Mobility. Houndmills: Palgrave Macmillan.

Morley, David. 2009. «For a Materialist, Non-Media-Centric Media Studies》. Television \& New Media 10 (1): 114-16.

Napoli, Philip M. 2010. Audience Evolution: New Technologies and the Transformation of Media Audiences. New York: Columbia University Press. 
Neumann-Braun, Klaus. 2005. «Strukturanalytische Rezeptionsforschung». In Qualitative Medienforschung : Ein Handbuch, herausgegeben von Lothar Mikos und Claudia Wegener, 58-66. Konstanz: UVK.

Ragnedda, Massimo, und Glenn W. Muschert. 2013. The Digital Divide: The Internet and Social Inequality in International Perspective. London: Routledge.

Ransom, Tailer G. 2017. «Process, habit, and flow: a phenomenological approach to material agency». Phenomenology and the Cognitive Sciences 18 (1): 19-37. https://doi.org/10.1007/s11097-017-9541-z.

Reinmann, Gabi. 2017. «Design Based Research». In Gestaltungsorientierte Forschung - Basis für soziale Innovationen. Erprobte Ansätze im Zusammenwirken von Wissenschaft und Praxis, herausgegeben von Dorothea Schemme und Hermann Novak, 49-61. Bielefeld: W. Bertelsmann.

Ren, Carina. 2010. «Non-Human Agency, Radical Ontology and Tourism Realities». Annals of Tourism Research 38 (3): 858-81.

Renckstorf, Karsten, und Fred Wester. 2001. «The «Media Use as Social Action> Approach: Theory, Methodology, and Research Evidence So Far». Communications 24 (4): 389-420.

Schatzki, Theodore R., Karin Knorr-Cetina, und Eike von Savigny, Hrsg. 2001. The Practice Turn in Contemporary Theory. London: Routledge.

Schimank, Uwe. 2010. Handeln und Strukturen: Einführung in die akteurtheoretische Soziologie. 4. Aufl. Weinheim et al.: Juventa-Verlag.

Schlör, Katrin. 2017. «Subjektorientierte medienpädagogische Forschung mit Familien in belasteten Lebenslagen - Chancen und Herausforderungen visueller Forschungsmethoden». Forschungswerkstatt Medienpädagogik. Projekt - Theorie - Methode. Zugriff 11.02.2019. http:// publ.forschungswerkstatt-medienpaedagogik.de/i/user/34.

Schorb, Bernd. 2011. «Zur Theorie der Medienpädagogik». In Medienbildung und Medienkompetenz: Beiträge zu Schlüsselbegriffen der Medienpädagogik, herausgegeben von Heinz Moser, Petra Grell, und Horst Niesyto, 81-94. München: kopaed.

Schutz, Alfred. 1967. The Phenomenology of the Social World. St. Evanston, IL: Northwestern University Press.

Selwyn, Neil. 2013. Distrusting Educational Technology: Critical Questions for Changing Times. London: Routledge.

Sewell, William H. 1992. «A Theory of Structure: Duality, Agency, and Transformation». American Journal of Sociology 98 (1): 1-29.

Shibutani, Tamotsu. 1955. «Reference Groups as Perspectives». American Journal of Sociology 60 (6): 562-69. 
Silverstone, Roger. 2006. «Domesticating Domestication. Reflections on the Life of a Concept». In Domestication of Media and Technology, herausgegeben von Thomas Berker, Maren Hartmann, Yves Punie, und Katie J. Ward, 229-48. London: Open University Press.

Strauss, Anselm L. 1978. «A Social Worlds Perspective». Studies in Symbolic Interaction 1: 119-28.

Strübing, Jörg. 2018. «Situationsanalyse: Eine pragmatische Erweiterung der Grounded Theory unter dem Eindruck der Postmoderne». In Handbuch Interpretativ forschen, herausgegeben von Leila Akremi, Nina Baur, Hubert Knoblauch, und Boris Traue, 681-706. Weinheim/ Basel: Beltz Juventa.

Suchman, Lucy A. 2007. Human-Machine Reconfigurations: Plans and Situated Actions. 2. Aufl. Cambridge MA: Cambridge University Press.

Teichert, Will. 1972. «〈Fernsehen〉 als soziales Handeln. Zur Situation der Rezipientenforschung: Ansätze und Kritik». Rundfunk und Fernsehen 20 (4): 421-39.

Thomas, Douglas, und John S. Brown. 2011. A New Culture of Learning: Cultivating the Imagination for a World of Constant Change. Lexington KY: CreateSpace Independent Publishing Platform.

Thompson, John B. 1995. Media and Modernity: A Social Theory of the Media. Cambridge: Cambridge University Press.

Tulodziecki, Gerhard. 2007. «Handlungs- und entwicklungsorientierte Medienpädagogik - theoretische Grundlagen, Umsetzungen und Forschung». In Jahrbuch Medien-Pädagogik 6: Medienpädagogik. Standortbestimmung einer erziehungswissenschaftlichen Disziplin, herausgegeben von Werner Sesink, Michael Kerres, und Heinz Moser, 102-17. Wiesbaden: VS Verlag für Sozialwissenschaften. https://oi. org/10.1007/978-3-531-90544-0_5.

Wolf, Karsten D. 2015. «Bildungspotenziale von Erklärvideos und Tutorials auf YouTube. Audiovisuelle Enzyklopädie, adressatengerechtes Bildungsfernsehen, Lehr-Lern-Strategie oder partizipative Peer Education?» merz | Medien + Erziehung 59 (1): 30-36.

Wolf, Karsten D., und Andreas Breiter. 2014. «Integration informeller und formaler Bildungsprozesse zur beruflichen Orientierung von Jugendlichen am Beispiel draufhaber.tv». In Lernen im Web 2.0 - Erfahrungen aus Berufsbildung und Studium, herausgegeben von Ulrich H. Hoppe, Nicole C. Krämer, Tina Ganster, Nicole Sträfling, und Nils Malzahn, 85-101. Bonn: BIBB. 
Wolf, Karsten D., Klaus Rummler, und Wibke Duwe. 2011. «Medienbildung als Prozess der Unsgestaltung zwischen formaler Medienerziehung und informeller Medienaneignung». In Medienbildung und Medienkompetenz: Beiträge zu Schlüsselbegriffen der Medienpädagogik, herausgegeben von Heinz Moser, Petra Grell, und Horst Niesyto, 137-58. München: kopaed.

Wolf, Karsten D., und Urzula Wudarski. 2017. «Communicative Figurations of Expertization: DIY_MAKER and Multi-Player Online Gaming (MOG) as Cultures of Amateur Learning». In Communicative Figurations: Transforming Communications in Times of Deep Mediatization, herausgegeben von Andreas Hepp, Uwe Hasebrink, und Andreas Breiter, 123-49. Cham: Palgrave Macmillan/Springer Nature. 